\title{
Co-occurrence of snow leopard Panthera uncia, Siberian ibex Capra sibirica and livestock: potential relationships and effects
}

\author{
Francesco Rovero, Claudio Augugliaro, Rasmus Worsøe Havmøller \\ Claudio Groff, Fridolin Zimmermann \\ Valentina OBERosLer and Simone Tenan
}

\begin{abstract}
Understanding the impact of livestock on native wildlife is of increasing conservation relevance. For the Vulnerable snow leopard Panthera uncia, wild prey reduction, intensifying human-wildlife conflicts and retaliatory killings are severe threats potentially exacerbated by the presence of livestock. Elucidating patterns of co-occurrence of snow leopards, wild ungulate prey, and livestock, can be used to assess the compatibility of pastoralism with conservation. We used camera trapping to study the interactions of livestock, Siberian ibex Capra sibirica and snow leopards in a national park in the Altai mountains, Mongolia. We obtained 494 detections of wild mammals and 912 of domestic ungulates, dogs and humans. Snow leopards and Siberian ibex were recorded 14 and 33 times, respectively. Co-occurrence modelling showed that livestock had a higher estimated occupancy (0.65) than ibex, whose occupancy was lower in the presence of livestock (o.11) than in its absence (0.34-0.35 depending on scenarios modelled). Snow leopard occupancy did not appear to be affected by the presence of livestock or ibex but the robustness of such inference was limited by uncertainty around the estimates. Although our sampling at presumed snow leopard passing sites may have led to fewer ibex detections, results indicate that livestock may displace wild ungulates, but may not directly affect the occurrence of snow leopards. Snow leopards could still be threatened by livestock, as overstocking can trigger human-carnivore conflicts and hamper the conservation
\end{abstract}

Francesco Rovero (Corresponding author) and Valentina Oberosler Tropical Biodiversity Section, MUSE - Museo delle Scienze, Corso del Lavoro e della Scienza 3, 38122 Trento, Italy. E-mail francesco.rovero@muse.it

Claudio Augugliaro Department of Ecology and Evolution, University of Lausanne, Lausanne, Switzerland

Rasmus Worsøe Havmøller Centre for Macroecology, Evolution and Climate, Natural History Museum of Denmark, University of Copenhagen, Copenhagen, Denmark

Claudio Groff Servizio Foreste e Fauna, Provincia Autonoma di Trento, Trento, Italy

Fridolin Zimmermann KORA, Muri, Switzerland

Simone Tenan Vertebrate Zoology Section, MUSE - Museo delle Scienze, Trento, Italy

Received 1 September 2017. Revision requested 24 October 2017.

Accepted 3 November 2017. First published online 4 March 2018. of large carnivores. Further research is needed to assess the generality and strength of our results.

Keywords Altai mountains, camera trapping, human-wildlife conflict, Mongolia, occupancy, occurrence interactions, Siberian ibex, snow leopard

Supplementary material for this article is available at https://doi.org/10.1017/Soo30605317001685

\section{Introduction}

The impact of livestock on native wildlife is a global conI servation issue (Prins, 1992; Fleischner, 1994; Berger et al., 2013) that remains poorly understood despite much debate (e.g. Ekernas et al., 2017). High densities of livestock induce competitive exclusion, resulting in decline of wild herbivores (Bagchi et al., 2004; Madhusudan, 2004), and pastoralism affects large carnivores (Ogutu et al., 2005; Ripple et al., 2014). In areas such as Central Asia, where numbers of free-ranging livestock have increased exponentially (Berger et al., 2013), including within protected areas (Bagchi et al., 2004), the cascading threats to large carnivores such as snow leopard Panthera uncia and wolf Canis lupus are of concern. These include the intensification of human-carnivore conflict caused by depredation, leading to the retaliatory killing of carnivores, and the reduction of wild herbivore prey (Treves \& Karanth, 2003; Mishra et al., 2010; Sharma et al., 2015). A recent study in Mongolia's Gobi steppe shows the complexity of the cascading effects of pastoralism on wildlife (Ekernas et al., 2017). Understanding the spatial interactions among large carnivores, wild herbivores and livestock is therefore of relevance for planning sustainable livestock grazing regimes and reducing threats.

Here we focus on co-occurrence patterns of snow leopards, one of their main wild prey, the Siberian ibex Capra sibirica, and domestic ungulates in a protected area in north-western Mongolia. Snow leopards have a vast but fragmented distribution across central Asia's mountain landscape, and their survival depends primarily on wild ungulates (Jackson et al., 2008; Snow Leopard Network, 2014). Globalization of the cashmere wool trade has increased livestock numbers across the snow leopard range, particularly in China and Mongolia, exposing this felid to intensified threats (Berger et al., 2013; 
Sharma et al., 2015). Prey reduction and human-wildlife conflicts resulting in the killing of snow leopards are the primary threats caused by livestock presence (Snow Leopard Network, 2014). Conflicts generated by depredation of livestock ( $\mathrm{Li}$ et al., 2013; Chen et al., 2016), patterns of predation on livestock and wild prey (Johansson et al., 2015), and the impact of livestock on snow leopard prey (Bagchi et al., 2004) have been assessed. The impact of livestock on snow leopards, however, remains less understood. There is evidence of increased intensity in snow leopard site use with increasing livestock numbers, with a possible decrease beyond a threshold of livestock densities (Sharma et al., 2015). Greater predation on livestock by snow leopards has been reported in areas with lower abundance of wild prey (Bagchi \& Mishra, 2006), although a review of 16 studies indicates that the proportions of wild ungulates and livestock in the diet of snow leopards are not correlated (Lovari et al., 2013).

We used camera trapping data and an occupancy framework (MacKenzie et al., 2002) to study the spatial interactions among snow leopards, ibex and livestock. The efficiency of camera trapping to detect snow leopards has been widely tested (Janečka et al., 2011) and recent studies show the potential of site occupancy modelling to study patterns in their distribution (Alexander et al., 2016a, b). We assess the usefulness of this approach to evaluate cooccurrence patterns of Siberian ibex, snow leopards and livestock by using an occupancy model that explicitly assesses co-occurrence (Waddle et al., 2010).

\section{Study area}

We conducted the study in the Siilkhem National Park, Part B (centred on $49^{\circ} 49^{\prime} 21.42^{\prime \prime} \mathrm{N}, 89^{\circ} 44^{\prime} 56.84^{\prime \prime} \mathrm{E} ; 1,400 \mathrm{~km}^{2}$ ), in the Altai Mountains, north-western Mongolia (Fig. 1), a steep, rocky and dry mountain environment up to 3,965 $\mathrm{m}$ altitude.
The study area is mainly grassland with sparse growth of shrubs on the slopes and larch Larix sibirica along valley bottoms. Traditional livestock husbandry is permitted in the limited use zones of national parks (Government of Mongolia, 1994), and most of the local people are pastoralists. There are large numbers of livestock: shepherds with dogs herd during the day along valleys and on accessible slopes, and livestock is housed in corrals at night. In the study district the number of goats increased from 21,937 in 1970 to 105,376 in 2015, with similar increases in other domestic animals (Mongolian Statistical Information Service, 2018).

\section{Methods}

\section{Data collection}

From March to June 2015 we used 49 camera traps across $513 \mathrm{~km}^{2}$ (the minimum convex polygon around the cameratrap sites). We originally designed a regular grid of camera traps, with cells of $2 \mathrm{~km}^{2}$ over the entire central and northern part of the Park, which, based on preliminary information, was considered suitable for snow leopards. Such spacing ensured that at least three camera traps sampled a snow leopard home range of c. 11-142 km ${ }^{2}$ (Jackson, 1996; McCarthy et al., 2005). After taking into account the area's topography and presumed snow cover, we reduced the extent of the grid to survey valleys and slopes that we could reach, up to $3,200 \mathrm{~m}$. We placed camera traps in each cell of this grid, on the nearest site to the centre of the grid cell suitable for snow leopard passage based on signs such as scrapes, scats, scratches on trees, scent markings and tracks. In the absence of such signs, we placed camera traps along probable snow leopard passing sites such as narrow valley bottoms and saddles along ridges. This was motivated by the fact that the snow leopard is the rarest

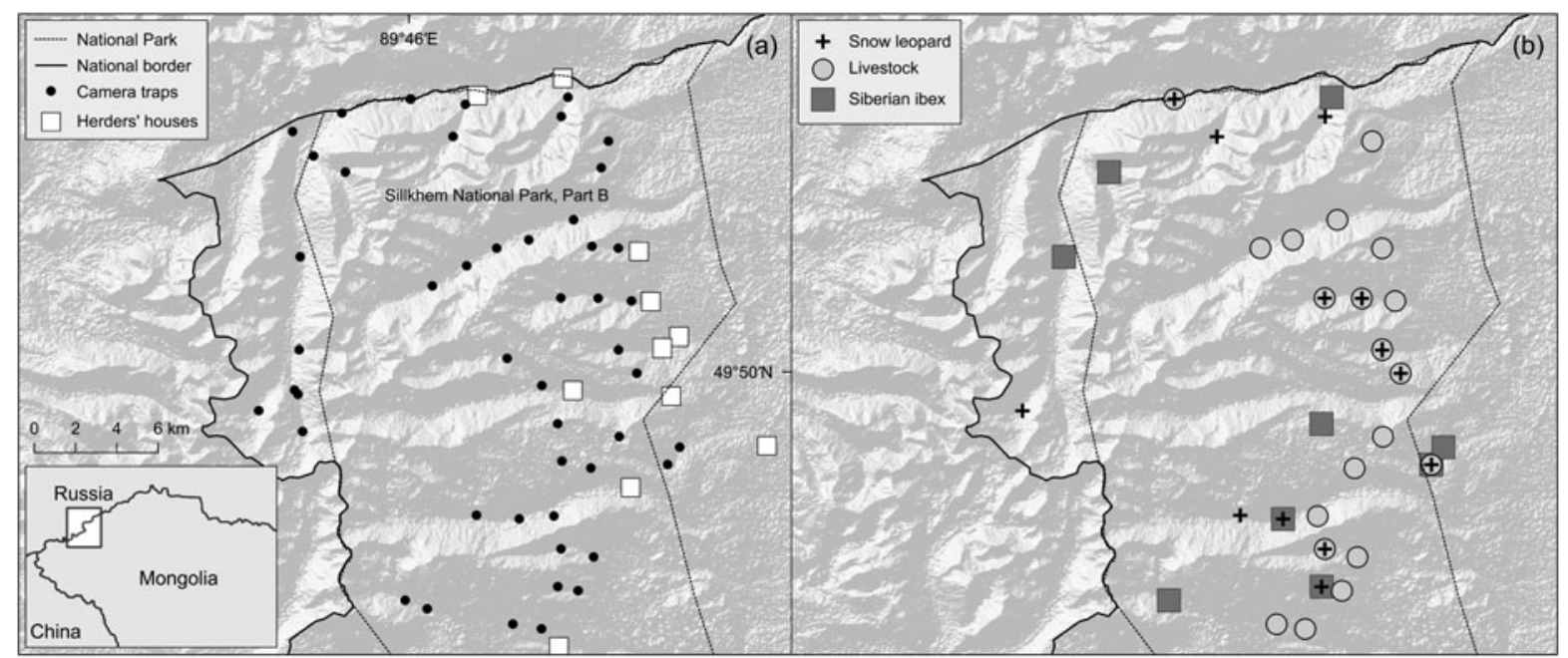

Fig. 1 The study area within Siilkhem National Park, Part B, north-western Mongolia: (a) locations of the 49 camera traps and herders' houses and camps; (b) sites where we detected livestock, Siberian ibex and snow leopards. 
and most elusive of the species we wanted to detect. Sightings of ibex and livestock indicated that the sites were also suitable to capture their presence, although this design may have favoured the detection of snow leopards over ibex and livestock. The selected sites (Fig. 1) were at 2,219-3,126 $\mathrm{m}$ altitude.

For logistical reasons we used two grids: the first $(26$ sites) sampled from 17 March to 4 May and the second (23 sites) from 5 May to 29 June 2015. We used Xenon white flash digital camera traps (Cuddeback Ambush, Non Typical Inc., Green Bay, USA), which take colour images at day and night with a trigger time of $0.25 \mathrm{~s}$. We set camera traps to work in continuous mode and fitted them in wooden bases on small rock piles c. $50 \mathrm{~cm}$ above the ground.

\section{Statistical analysis}

We processed data in $R$ (R Development Core Team, 2015), and first derived species' raw descriptors of captures: independent events (using a $15 \mathrm{~min}$ interval to separate subsequent images of the same species), relative abundance index (the ratio of events to sampling effort) and naïve occupancy (Rovero et al., 2014). For snow leopards, ibex and domestic ungulates we built matrices of detection/nondetection on daily occasions and used a model for estimating co-occurrence of interacting species developed by Waddle et al. (2010). Occurrence and detectability were modelled for a single dominant species and one or more subordinate species, where probabilities referred to subordinate species are conditional on presence of the dominant one. Given the limited number of snow leopard and ibex detections, we fitted models by contrasting two scenarios in which livestock occurrence was assumed independent of the other two species: livestock presence determines the occurrence of both snow leopard and ibex (scenario 1); livestock presence determines the occurrence of ibex and, in turn, ibex occurrence determines snow leopard occurrence (scenario 2). Sample sizes limited the number of environmental and human disturbance factors that we could use as model covariates. Thus, we considered (1) elevation at the camera trap site, derived from a Digital Elevation Model, as a proxy for the variation in habitat within the elevation range surveyed, and (2) distance of camera-trap sites to occupied herders' houses and camps (Fig. 1), as a proxy for anthropogenic disturbance.

Detailed model formulation and parameter constraints, and model fitting procedures, are reported in Supplementary Material 1. Livestock occurrence probability was modelled as a function of elevation and distance to herders' houses. We assumed ibex occurrence depends on the occurrence of livestock and distance to herders' houses. Livestock encounter probability was assumed to be constant and independent of occurrence of other species. Ibex encounter probability was tested for an effect of distance to herders' houses and camps. Snow leopard encounter probability was a function of distance to herders' houses and camps, as we assumed these places may influence the elusiveness of snow leopards. Models were fitted using the Markov chain Monte Carlo framework, implemented in JAGS (Plummer, 2003), which we executed from $R$ (see Supplementary Material 2 for model code and Supplementary Figs. 1 \& 2 for the Bayesian learning plots of the posterior distributions).

\section{Results}

Total sampling effort was 2,225 camera days (mean $45.4 \pm$ SD 6.6 per camera) from the 49 camera traps set, yielding 494 detections of 12 species of wild mammals, 105 of people and 367 of livestock, primarily goats and sheep (188) and cattle/yaks (163). Livestock was detected at 21 locations (naïve occupancy $=0.43$; Supplementary Table 1), with herds of sheep and goats numbering in their hundreds. We obtained 17 photographs of snow leopards representing 14 events at 13 locations (naïve occupancy $=0.27$ ). By comparing individual coat patterns across events, we concluded that three individual leopards were detected, with all photographs allowing for identification. The sex of these individuals could not be determined from the photographs. We also recorded 33 events of Siberian ibex at nine locations (naïve occupancy $=0.18)($ Fig. 1). The group size of ibex, as determined from camera trap images and opportunistic sightings, was 1-31. Detections did not show any marked pattern of temporal segregation: livestock and ibex were predominantly diurnal although ibex was occasionally detected in the first hours after dusk; snow leopards were active during 5.00-12.00 and the few detections did not reveal any peak of activity.

Occupancy modelling showed that for both scenarios livestock had a higher mean estimated occupancy (mean = 0.65; 95\% credible interval $0.44-0.83$; Table 1 ) than ibex, and ibex occupancy was negatively affected by the presence of livestock. The decrease in mean point estimate of ibex occupancy varied from 0.34 to 0.11 for scenario 1 and from 0.35 to 0.11 for scenario 2 , in the absence and presence of livestock, respectively (Fig. 2, Table 1). In contrast, in the first scenario the mean point estimate of snow leopard occupancy was not affected by the presence of livestock ( 0.77 and 0.74 with and without livestock, respectively; Fig. 2), and similarly for detectability, which was 0.01 in the presence (95\% credible interval o.004-0.022) and absence (95\% credible interval 0.003-0.021) of livestock (Table 1). Similarly, in the second scenario, the occupancy of snow leopard was not affected by the presence of ibex (mean = 0.80, $95 \%$ credible interval $0.340-0.996$, and mean $=0.76$, $95 \%$ credible interval $0.295-0.994$, in the presence and 
TABLE 1 Posterior parameter estimates (mean \pm SD, and 95\% credible interval quantiles, CRI) for occupancy $(\psi)$ and detection probability $(p)$ in the two scenarios (scenario 1: livestock presence determines the occurrence of both snow leopard Panthera uncia and ibex Capra sibirica; scenario 2: livestock presence determines the occurrence of ibex and, in turn, ibex occurrence determines snow leopard occurrence.

\begin{tabular}{|c|c|c|c|c|}
\hline \multirow[b]{2}{*}{ Parameter ${ }^{*}$} & \multicolumn{2}{|l|}{ Scenario 1} & \multicolumn{2}{|l|}{ Scenario 2} \\
\hline & Mean \pm SD & 95\% CRI & Mean \pm SD & 95\% CRI \\
\hline \multicolumn{5}{|l|}{ Occurrence } \\
\hline $\bar{\Psi}^{\mathrm{LI}}$ & $0.649 \pm 0.100$ & $0.443-0.830$ & $0.648 \pm 0.100$ & $0.445-0.830$ \\
\hline $\bar{\Psi}^{\mathrm{IB} \mid \mathrm{LI}}$ & $0.105 \pm 0.074$ & $0.013-0.292$ & $0.106 \pm 0.074$ & $0.013-0.292$ \\
\hline $\bar{\Psi}^{\mathrm{IB} \mid \overline{\mathrm{LI}}}$ & $0.341 \pm 0.123$ & $0.156-0.646$ & $0.346 \pm 0.128$ & $0.155-0.679$ \\
\hline $\bar{\Psi}^{\mathrm{SL} / \mathrm{LI}}$ & $0.771 \pm 0.184$ & $0.345-0.995$ & & \\
\hline $\bar{\Psi}^{\mathrm{SL} \mid \overline{\mathrm{LI}}}$ & $0.736 \pm 0.203$ & $0.281-0.992$ & & \\
\hline $\bar{\Psi}^{\mathrm{SL} \mid \mathrm{IB}}$ & & & $0.796 \pm 0.183$ & $0.340-0.996$ \\
\hline $\bar{\Psi}^{\mathrm{SL} \mid \overline{\mathrm{IB}}}$ & & & $0.758 \pm 0.197$ & $0.295-0.994$ \\
\hline \multicolumn{5}{|l|}{ Detectability } \\
\hline $\bar{p}^{\mathrm{LI}}$ & $0.165 \pm 0.012$ & $0.142-0.189$ & $0.165 \pm 0.012$ & $0.142-0.188$ \\
\hline $\bar{p}^{\mathrm{IB}}$ & $0.057 \pm 0.013$ & $0.034-0.084$ & $0.057 \pm 0.013$ & $0.004-0.016$ \\
\hline $\bar{p}^{\mathrm{SL}}$ & & & $0.009 \pm 0.003$ & $0.004-0.016$ \\
\hline $\bar{p}^{\mathrm{SL} \mid \mathrm{LI}}$ & $0.010 \pm 0.005$ & $0.004-0.022$ & & \\
\hline $\bar{p}^{\mathrm{SL} \mid \overline{\mathrm{LI}}}$ & $0.010 \pm 0.005$ & $0.003-0.021$ & & \\
\hline \multicolumn{5}{|c|}{ Effect of covariates } \\
\hline$\beta_{\text {elev }}^{\mathrm{LI}}$ & $-2.189 \pm 0.721$ & $-3.777--0.943$ & $-1.190 \pm 0.718$ & $-3.768--0.965$ \\
\hline$\beta_{\text {dist }}^{\text {elev }}$ & $-0.850 \pm 0.715$ & $-2.291-0.529$ & $-0.858 \pm 0.711$ & $-2.311-0.510$ \\
\hline$\beta_{\text {elev }}^{\mathrm{SL}}$ & $-0.214 \pm 1.670$ & $-3.258-3.553$ & $-0.311 \pm 1.702$ & $-3.307-3.475$ \\
\hline$\beta_{\text {dist }}^{\text {SL }}$ & $-0.279 \pm 1.552$ & $-3.191-3.186$ & $-0.280 \pm 1.630$ & $-3.288-3.157$ \\
\hline$\alpha_{\mathrm{dist}}^{\mathrm{dilst}}$ & $-0.273 \pm 0.448$ & $-1.174-0.578$ & $-0.350 \pm 0.449$ & $-1.283-0.493$ \\
\hline$\beta_{\text {dist }}^{\text {dist }}$ & $0.569 \pm 0.780$ & $-0.427-2.729$ & $0.618 \pm 0.840$ & $-0.408-2.953$ \\
\hline$\alpha_{\text {dist }}^{1 \mathrm{IIt}}$ & $-0.158 \pm 0.265$ & $-0.697-0.336$ & $-0.174 \pm 0.269$ & $-0.711-0.328$ \\
\hline
\end{tabular}

(a) Scenario 1

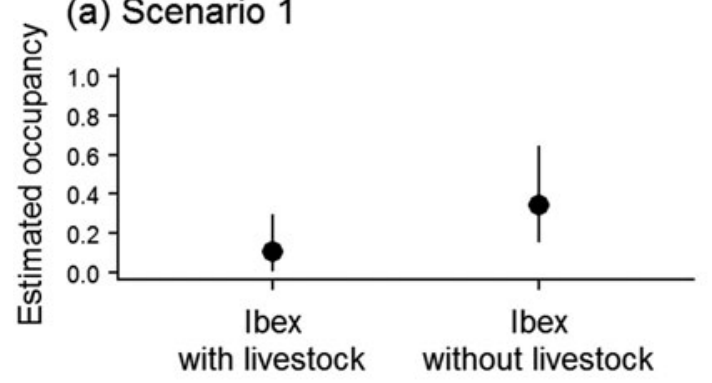

(c) Scenario 1

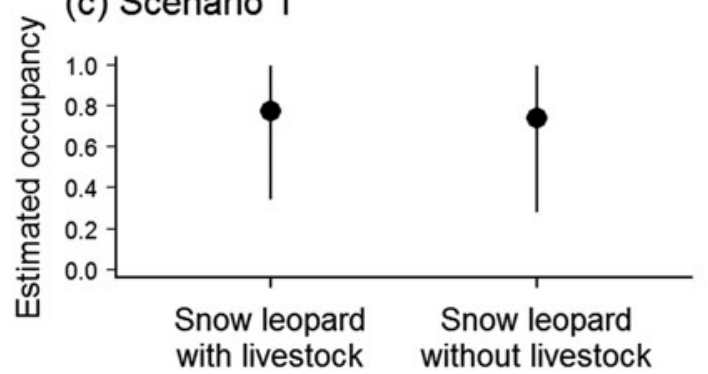

(b) Scenario 2

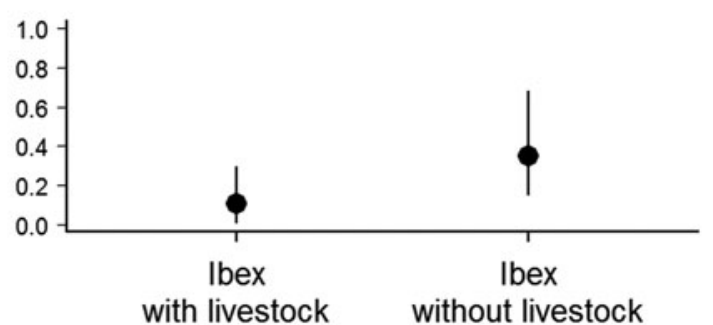

(d) Scenario 2

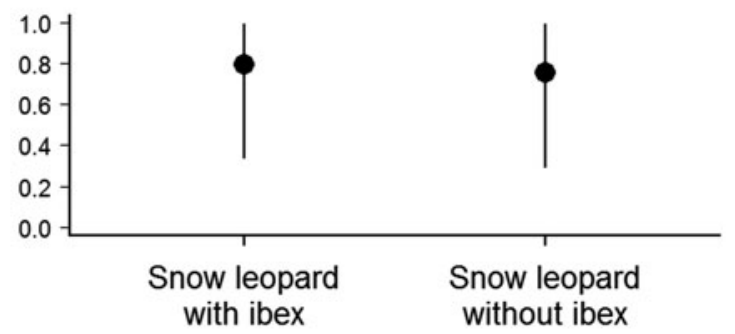

FIG. 2 Occupancy probability (mean and 95\% credible interval) estimated from the modelling of co-occurrence patterns of (a) ibex depending on livestock, scenario 1; (b) ibex depending on livestock, scenario 2; (c) snow leopard depending on livestock, scenario 1; and (d) snow leopard depending on ibex, scenario 2. 
absence of ibex, respectively). The only covariate with a significant impact (i.e. $95 \%$ credible interval did not encompass zero) was site elevation, which had a negative effect on the estimated occupancy of livestock in both scenarios (Table 1).

\section{Discussion}

Our analysis suggests three patterns: (1) the occurrence of livestock may have a negative effect on the occurrence of ibex, with a difference in the mean point estimates despite some overlap in credible intervals; (2) livestock presence did not appear to affect the occurrence of snow leopards; (3) snow leopard occurrence did not seem to be affected by the presence of ibex. The small number of snow leopard and ibex detections limited the precision of our estimates, suggesting caution about the generality of the results. We acknowledge that the study design we used, primarily targeted at the snow leopard, may have resulted in an undersampling bias for ibex and livestock. Additional data on herd numbers and movement patterns for livestock and ibex would be required to further assess these interactions.

Although the study area is a national park, our camera traps detected over 10 times more livestock compared to the only wild ungulate recorded, the Siberian ibex. Although we detected livestock at $43 \%$ of recording sites, our model estimated that livestock would occur at over two thirds of sites (mean estimated occupancy $=0.65$ ). Data on temporal trends in the abundance of the ibex population will be required to assess whether the co-occurrence pattern we found reflects declining abundance of wild herbivores with increasing livestock densities, as suggested by studies in the Trans-Himalaya (Mishra et al., 2002; Bagchi et al., 2004).

There were 14 detections of three snow leopards at remote sites and larger valleys heavily used by livestock, and both snow leopards and livestock were detected at seven sites (Fig. 1). Beyond a threshold of livestock density, reduced wild prey populations and associated human disturbance may negatively influence the intensity of snow leopard habitat use (Sharma et al., 2015). In the Tost mountains, southern Mongolia, the snow leopard diet included more wild prey $(73 \%)$ than livestock (27\%; Johansson et al., 2015), despite livestock abundance being one order of magnitude higher. Data on retaliatory killings and predation pattern will be necessary to further examine how livestock affects snow leopards. Our discussions with local herders indicate that predation of free-ranging livestock occurs regularly, but this was attributed primarily to wolves. Predation in corrals was reported to occur sporadically, as in the Tost mountains (Johansson et al., 2015). Shepherds appeared aware that unlike wolves, snow leopards are protected, and their attitude towards snow leopards seemed more positive than towards wolves. We found skins of wolves in some herders' houses, but no evidence of snow leopard poaching.
These observations indicate that although livestock is widespread in Part B of Siilkhem National Park, and could have a negative impact on the occurrence of ibex, it may not have a significant impact on the occurrence of snow leopards, and there is no evidence of intense conflicts with local herders.

At a large scale there is generally a positive association between wild prey presence and site use by snow leopards (McCarthy et al., 2005; Alexander et al., 2016b) but at the local scale this pattern may not hold. In China site use by snow leopards was not strongly associated with the presence of bharal Pseudois nayaur, suggesting this pattern may be a result of the lack of spatial heterogeneity in bharal distribution, which were detected at all sites (Alexander et al., 2016a). We did not find a pattern of association, as the occurrence of snow leopards did not vary with the presence or absence of ibex. This result is compatible with the hypothesis that predator-prey occurrences may be decoupled (Ekernas et al., 2017), although the possible under-sampling of ibex may have masked the pattern of association.

In conclusion, our results support the potential of the occupancy framework for analysing snow leopard detection/ non-detection data from camera traps (Alexander et al., 2016a). An improved evaluation of its application to model co-occurrence patterns will require greater sample sizes, and ideally site selection should not bias detection of a particular species. The added advantage of the model we used is that interactions among species are modelled explicitly, along with the effect of environmental covariates. The main constraint was the small number of detections of snow leopards and ibex. This limited the number of parameters and hence the co-occurrence interactions and covariate effects that we could model simultaneously.

The indication that livestock may negatively impact wild ungulates merits further research, as does the result that livestock may not directly affect the occurrence of the snow leopard. The negative effects that depletion of wild prey may have on large carnivores are well known, as is the potential resulting escalation of human-wildlife conflicts (Berger et al., 2013; Ekernas et al., 2017). Given that the study area is a national park, we suggest that ensuring protection of Siberian ibex and snow leopards in the Park requires a revision of the livestock grazing zone, and increased law enforcement, with emphasis on the creation and protection of livestock-free areas. Conservation efforts should concomitantly target livestock practices by engaging herders in the process of developing new grazing plans, as elaborated in the Snow Leopard Survival Strategy (Snow Leopard Network, 2014).

\section{Acknowledgements}

We thank Sandro Lovari and an anonymous reviewer for constructive comments, and Justine Alexander, Örian 
Johansson and Miha Krofel for their input. The study was funded by MUSE - Museo delle Scienze (Italy) and Green Initiative (Mongolia), with a contribution from the Natural History Museum of Denmark. RWH acknowledges funding from the Danish National Research Foundation to the Center for Macroecology, Evolution and Climate (grant no. DNRF96). Fototrappolaggio provided some of the camera traps. The Ministry of Environment and Tourism of Mongolia and the Altai Mountain Protected Area Authority provided research permits and support. We thank Jukhan Mergenbay, Khairat Amangeldi and Veronika Jahn for valuable field assistance, and Choikhand Janchivlamdan of Green Initiative for logistic support. Asja Marchetto helped annotate images. Aaron Iemma and Paolo Pedrini helped with mapping and logistics.

\section{Author contributions}

FR, CA and ST conceived and designed the study; FR, CA, FZ, RGH and CG collected the data; FR, ST and VO analysed the data. All authors, led by FR, contributed to the writing of the manuscript.

\section{References}

Alexander, J.S., Shi, K., Tallents, L.A. \& Riordan, P. (2016a) On the high trail: examining determinants of site use by the Endangered snow leopard Panthera uncia in Qilianshan, China. Oryx, 50, 231238.

Alexander, J.S., Gopalaswamy, A.M., Shi, K., Hughes, J. \& Riordan, P. (2016b) Patterns of snow leopard site use in an increasingly human-dominated landscape. PLoS ONE, 11, e0155309.

BAGCHI, S. \& Mishra, C. (2006) Living with large carnivores: predation on livestock by the snow leopard (Uncia uncia). Journal of Zoology, 268, 217-224.

Bagchi, S., Mishra, C. \& Bhatnagar, Y.V. (2004) Conflicts between traditional pastoralism and conservation of Himalayan ibex (Capra sibirica) in the trans-Himalayan mountains. Animal Conservation, 7, 121-128.

Berger, J., Buuveibaatar, B. \& Mishra, C. (2013) Globalization of the cashmere market and the decline of large mammals in Central Asia. Conservation Biology, 27, 679-689.

Chen, P., Gao, Y., Lee, A.T.L., Cering, L., Shi, K. \& Clark, S.G. (2016) Human-carnivore coexistence in Qomolangma (Mt. Everest) Nature Reserve, China: patterns and compensation. Biological Conservation, 197, 18-26.

Ekernas, L.S., Sarmento, W.M., Davie, H.S., Reading, R.P., Murdoch, J., Wingard, G.J. et al. (2017) Desert pastoralists' negative and positive effects on rare wildlife in the Gobi. Conservation Biology, 31, 269-277.

Fleischner, T.L. (1994) Ecological costs of livestock grazing in western North America. Conservation Biology, 8, 629-644.

Government of Mongolia (1994) Law on special protected areas. Government of Mongolia, Ulaanbaatar, Mongolia. Http:// extwprlegsi.fao.org/docs/pdf/mon77268E.pdf [accessed 16 January 2018].
Jackson, R., Mallon, D., McCarthy, T., Chundawat, R.A. \& Haвiв, B. (2008) Panthera uncia. In The IUCN Red List of Threatened Species, Version 2014. Http://www.iucnredlist.org. JACKSON, R.M. (1996) Home Range, Movements and Habitat Use of Snow Leopard (Uncia uncia) in Nepal. PhD thesis. University of London, UK.

JanečKa, J.E., Munkhtsog, B., Jackson, R.M., Naranbaatar, G., Mallon, D.P. \& Murphy, W.J. (2011) Comparison of noninvasive genetic and camera-trapping techniques for surveying snow leopards. Journal of Mammalogy, 92, 771-783.

Johansson, Ö., McCarthy, T., Samelius, G., Andrén, H., Tumursukh, L. \& Mishra, C. (2015) Snow leopard predation in a livestock dominated landscape in Mongolia. Biological Conservation, 184, 251-258.

Li, J., Yin, H., WANG, D., JiAGONG, Z. \& Lu, Z. (2013) Human-snow leopard conflicts in the Sanjiangyuan region of the Tibetan plateau. Biological Conservation, 166, 118-123.

Lovari, S., Ventimiglia, M. \& Minder, I. (2013) Food habits of two leopard species, competition, climate change and upper treeline: a way to the decrease of an endangered species? Ethology, Ecology \& Evolution, 25, 305-318.

MacKenzie, D.I., Nichols, J.D., Lachman, G.B., Droege, S., Andrew Royle, J. \& Langtimm, C.A. (2002) Estimating site occupancy rates when detection probabilities are less than one. Ecology, 83, 2248-2255.

Madhusudan, M.D. (2004) Recovery of wild large herbivores following livestock decline in a tropical Indian wildlife reserve. Journal of Applied Ecology, 41, 858-869.

McCarthy, T.M., Fuller, T.K. \& Munkhtsog, B. (2005) Movements and activities of snow leopards in Southwestern Mongolia. Biological Conservation, 124, 527-537.

Mishra, C., Bagchi, S., Namgail, T. \& Bhatnagar, Y.V. (2010) Multiple use of trans-Himalayan rangelands: reconciling human livelihoods with wildlife conservation. In Wild Rangelands: Conserving Wildlife While Maintaining Livestock in Semi-arid Ecosystems (eds J. Du Toit, R. Kock \& J. Deutsch), pp. 291-311. Blackwell Publishing, Oxford, UK.

Mishra, C., Van Wieren, S.E., HeitköNig, I.M.A. \& Prins, H.H.T. (2002) A theoretical analysis of competitive exclusion in a Trans-Himalayan large-herbivore assemblage. Animal Conservation, 5, 251-258.

Mongolian Statistical Information Service (2018) Social and economic situation of Mongolia. National Statistics Office of Mongolia. Http://1212.mn [accessed 18 January 2018].

Ogutu, J.O., Bhola, N. \& Reid, R. (2005) The effects of pastoralism and protection on the density and distribution of carnivores and their prey in the Mara ecosystem of Kenya. Journal of Zoology, 265, 281-293.

Plummer, M. (2003) JAGS: a program for analysis of Bayesian graphical models using Gibbs sampling. In Proceedings of the 3 rd International Workshop on Distributed Statistical Computing (eds K. Hornik, F. Leisch \& A. Zeileis). Technische Universität Wien, Vienna, Austria.

Prins, H.H.T. (1992) The pastoral road to extinction: competition between wildlife and traditional pastoralism in East Africa. Environmental Conservation, 19, 117-123.

R Development Core Team (2015) R: A Language and Environment for Statistical Computing. R Foundation for Statistical Computing, Vienna, Austria. Http://www.R-project.org [accessed 30 January 2018].

Ripple, W.J., Estes, J.A., Beschta, R.L., Wilmers, C.C., Ritchie, E.G., Hebblewhite, M. et al. (2014) Status and ecological effects of the world's largest carnivores. Science, 343, 1241484.

Rovero, F., Martin, E., Rosa, M., Ahumada, J.A. \& Spitale, D. (2014) Estimating species richness and modelling habitat 
preferences of tropical forest mammals from camera trap data. PLoS ONE, 9, e103300.

Sharma, R.K., Bhatnagar, Y.V. \& Mishra, C. (2015) Does livestock benefit or harm snow leopards? Biological Conservation, 190, 8-13.

SnOw Leopard Network (2014) Snow Leopard Survival Strategy. Revised 2014 Version, Snow Leopard Network, Seattle, Washington, USA. Http://www.snowleopardnetwork.org/docs/Snow_Leopard_ Survival_Strategy_2014.1.pdf [accessed 30 January 2018].

Treves, A. \& Karanth, K.U. (2003) Human-carnivore conflict and perspectives on carnivore management worldwide. Conservation Biology, 17, 1491-1499.

Waddle, J.H., Dorazio, R.M., Walls, S.C., Rice, K.G., Beauchamp, J., Schuman, M.J. \& Mazzotti, F.J. (2010) A new parameterization for estimating co-occurrence of interacting species. Ecological Applications, 20, 1467-1475.

\section{Biographical sketches}

Francesco Rovero is an ecologist and conservation scientist who focuses on ecological modelling of larger mammals, and uses camera trapping extensively. Claudio Augugliaro studies the ecology and conservation status of snow leopards. RASMU S HAV M ØLLER specializes in the ecology of large mammals and conservation, with expertise in genetics, camera trapping and GPS-tracking. Cla A dio Groff is an expert in large carnivores, focusing on human-carnivore conflicts and conservation management issues. Fridolin Zimmermann coordinates monitoring of large carnivores in Switzerland and has extensive expertise in camera trapping. VALENTINA OBERosler is an ecologist studying the distribution of larger mammals using systematic camera trapping coupled with occupancy modelling. SimONE TENAN is a quantitative ecologist who focuses on ecological applications in the general fields of population dynamics, community ecology, and conservation biology. 